Pedagogía y Saberes $n .^{\circ} 56$

Universidad Pedagógica Nacional

Facultad de Educación. 2022. pp. 103-115

\title{
Los primeros licenciados en Ciencias Biológicas en Colombia (1935-1950)*
}

\section{The First Teachers in Biological Sciences in Colombia (1935-1950) \\ Os primeiros professores em Ciências Biológicas na Colômbia (1935-1950)}

Deysi Serrato-Rodríguez ${ }^{* *}$

\section{Para citar este artículo}

Serrato-Rodríguez, D. (2022). Los primeros licenciados en Ciencias Biológicas en Colombia (1935-1950). Pedagogía y Saberes, (56). https://doi.org/10.17227/pys.num56-12348

* Este artículo de reflexión emerge de la tesis de investigación desarrollada en el marco del Doctorado Interinstitucional en Educación de la Universidad Pedagógica Nacional, titulada "La formación de maestros de Biología y la biología como saber escolar en Colombia: emergencias, transformaciones y desplazamientos".

** Magíster en Educación. Profesora, Universidad Pedagógica Nacional. Correo: dlserrator@pedagogica.edu. co. Orcid: orcid.org/ 0000-0002-9071-4589. 


\title{
Resumen
}

El presente artículo busca visibilizar algunas consideraciones y elementos ligados con la formación de maestros de Biología en Colombia, que se retoman del trabajo investigativo realizado en el marco del Doctorado Interinstitucional en Educación de la Universidad Pedagógica Nacional. A partir de un acercamiento histórico, se pretende comprender la confluencia de relaciones que han posibilitado la emergencia de los primeros licenciados en Ciencias Biológicas del país; así, cuestiones como la actualización en modernos métodos pedagógicos, el aumento de la población de maestros titulados para la atención de las regiones, el mejoramiento de las condiciones laborales, el posicionamiento de la ciencia como elemento clave de la modernización y la preocupación por la vida a propósito de su regulación, se constituyen en aspectos fundamentales para entender la relevancia de la biología en la época y la necesidad de contar con profesores que formen en dicho saber. Estas relaciones cobran importancia en la medida que permiten problematizar y cuestionar la formación de maestros de Biología en la actualidad, con la intención de poner bajo sospecha su posible desplazamiento debido a los pocos programas de formación que existen y a la mirada interdisciplinar que se instala desde las ciencias naturales, las ciencias de la vida o las ciencias complejas.

\section{Palabras clave}

enseñanza de la biología; formación de docentes; escuela; historia de la educación

\begin{abstract}
This article seeks to make visible some considerations and elements related to the training of Biology teachers in Colombia, which are taken from the research work carried out in the framework of the Interinstitutional Doctorate in Education of the Universidad Pedagógica Nacional. From a historical approach, the aim is to understand the relationships that have made possible the emergence of the first graduates in Biological Sciences in the country. Thus, issues such as the updating in modern pedagogical methods, the increase in the population of qualified teachers for the attention of the regions, the improvement of working conditions, the positioning of science as a key element of modernization, and the concern for life regarding its regulation, are fundamental aspects to understand the relevance of biology at the time and need to have teachers trained in this knowledge. These relationships become important to the extent because it allows us to problematize and question the training of biology teachers at present, to place under suspicion its possible displacement due to the few training programs that exist and the interdisciplinary view that is installed from the natural sciences, life sciences or complex sciences.
\end{abstract}

\section{Keywords}

biology education; teacher training; school; educational history

\section{Resumo}

Este artigo de reflexão procura tornar visível algumas considerações e elementos relativos à formação de professores de biologia na Colômbia, que fazem parte da tese elaborada no Doutorado Interinstitucional em Educação da Universidade Nacional Pedagógica. Desta forma, a partir de uma abordagem histórica, pretende-se compreender a confluência de relações que possibilitaram o surgimento dos primeiros graduados em biologia no país, portanto, questões como a atualização nos métodos pedagógicos modernos, o aumento da população de. professores qualificados para o atendimento das regiões, a melhoria das condições de trabalho, o posicionamento da ciência como elemento-chave da modernização e a preocupação pela vida na sua regulamentação, constituem-se como aspetos fundamentais para compreender a relevância da biologia e a necessidade de ter professores que se formem nesse saber. Essas relações são importantes porque permitem problematizar e questionar a formação dos professores de biologia na atualidade, com a intenção de colocar sob suspeita seu possível deslocamento devido aos poucos programas de formação existentes e à perspectiva interdisciplinar que se instala desde as Ciências Naturais, Ciências da Vida ou as Ciências Complexas.

\section{Palavras-chave}




\section{Consideraciones frente a la formación de maestros de Biología en Colombia: algunas problematizaciones}

Realizar una aproximación histórica a la formación de maestros de Biología no es un ejercicio que busque rescatar su pertinencia, ni marcar su punto de origen ni el recorrido de lo que debería haber sido desde la añoranza; por el contrario, es una problematización tendiente a visibilizar el presente de los procesos de preparación de los profesores de Biología, las tensiones que la atraviesan y sus posibles proyecciones. En esa medida, dicha formación no ha existido desde siempre; su emergencia puede ser rastreada alrededor de la primera mitad del siglo XX, a partir de los procesos de modernización, el posicionamiento de la ciencia y la preocupación alrededor de cuestiones básicas que atraviesan el bienestar de la población, tales como la alimentación, la salud, la raza y la pobreza, entre otros. Por tanto, la selección del periodo en el que se ubica esta reflexión responde a criterios de archivo que remiten a las primeras titulaciones de maestros en Ciencias Biológicas en Colombia.

Ahora bien, la lectura que se hace de la formación de maestros de Biología en la actualidad sitúa su posible desplazamiento ${ }^{1}$ por una serie de relaciones a propósito de enunciados como interdisciplinariedad y vida, además, del lugar que adquiere la biología escolar en la contemporaneidad y que podría estar relacionado con otros discursos como la educación ambiental, la educación sexual, la biotecnología, la biodiversidad, la tecnociencia, que terminan desbordando el discurso biológico y llevan a preguntarse por la pertinencia de una licenciatura en Biología en particular, y sitúan otras ópticas de la formación desde las ciencias naturales y la educación ambiental, las que

1 La noción de desplazamiento se constituye en una categoría a través de la cual se puede ver el proceso y, a la vez, los efectos y los movimientos de la pertinencia de unos saberes en el momento actual. Se trata de interrogar lo que hace que existan saberes que ocupen unos lugares donde otros no lo hacen, y parecen inhabitables o con espacios más reducidos. De acuerdo con ello, el desplazamiento expresa un movimiento, un cruce distinto de relaciones sobre el saber, pero no implica una total desaparición, sino unas condiciones de existencia diferentes en un momento histórico particular. En suma, es una pregunta por los modos de funcionamiento del poder sobre la formación de maestros de Biología en Colombia y de la enseñanza de dicha disciplina en la escuela. Por esto, resulta importante cuestionarse por las prácticas que generan condiciones favorables a la constitución de ciertos saberes, modos de pensar, hacer y ser. buscan brindar elementos para solventar problemas relevantes asociados a la vida de los sujetos, pero que es necesario cuestionar también.

La sospecha de dicho desplazamiento se relaciona, adicionalmente, con una apuesta desde las ciencias de la vida y las ciencias complejas en cuanto posibilidad para construir lecturas más articuladas y sistémicas sobre la vida en relación con lo vivo, donde la biología se ubica como la superficie que permite la construcción de encuentros con otras disciplinas. Así se encuentra que "una de las principales particularidades de la Biología hoy en día es precisamente su carácter transdisciplinario, su necesidad de dialogar y colaborar en [...] áreas fronterizas hasta hace poco inéditas, ocultas y, por ende, desatendidas" (Ruiz y Velázquez, 2014, p. 520), que remiten al tratamiento de los denominados problemas de frontera, entendidos como

aquellos que no pertenecen ya a una sola ciencia o disciplina sino, por el contrario, aquellos en los que confluyen modelos, explicaciones, lenguajes y tradiciones investigativas diferentes. Los problemas de frontera al mismo tiempo que dan lugar a nuevas ciencias de frontera, emergen como la base misma a partir de la cual son posibles síntesis de conocimientos. En resumen, un problema de frontera produce un desplazamiento de la mirada tradicional de una tradición investigativa y sienta las bases para nuevos paradigmas. (Maldonado, 2007, p. 71)

Así se abre un campo de posibilidad donde se sitúan otras formas de comprensión para atender aspectos como la crisis ambiental, el desarrollo de las tecnologías convergentes desde las NBIC (nano, bio, info, cogno), las implicaciones de la ingeniería genética, la importancia de la bioética, entre otros, que, a su vez, estarían posicionando los bioconceptos como espacio relacional para comprender distintas realidades, por cuanto estos "describen prácticas sociales que están reconfigurando la forma de estudiar y entender las ciencias de la vida" (Rodríguez, 2019, p. 203). Cada vez es más frecuente encontrar en funcionamiento nociones como biocapital, bioeconomía, biovalor, biohacking, bioarte y biogestión que están tejiendo otras relaciones entre la vida y lo vivo, y que hacen un llamado de atención al escenario educativo toda vez que es necesario transformar los procesos de formación a la luz de estas nuevas discusiones.

Otro aspecto que entra en juego a propósito del posible desplazamiento es la disminución de programas de formación de maestros de Biología. Desde una perspectiva histórica, hacia 1981 se registraba alrededor de veinte programas en distintas universi- 
dades en comparación con cuatro licenciaturas que existen actualmente a nivel nacional en la Universidad Pedagógica Nacional, la Universidad Francisco José de Caldas, la Universidad Santo Tomás y la Universidad de los Andes. Esta disminución también tiene que ver con el alto número de licenciaturas en Ciencias Naturales y Educación Ambiental que se ofrecen en el resto del país, y que tienen como característica una visión más integral y funcional del saber.

Por consiguiente, se justifica la pertinencia de formar docentes con capacidad para identificar, analizar y resolver los problemas de su ambiente y de su sociedad, buscando desarrollar niveles de conciencia cada vez más elevados en quienes participan y hacen parte de los procesos que destaquen no solo el "problema" sino que permitan determinar cuál es la actitud al respecto (Universidad del Tolima, 2016). Desde estos presupuestos, se encuentra que los programas de Ciencias Naturales permiten "ofrecer una amplia gama de posibilidades de movilización, convenios y espacios de formación; además, asegurar el aprendizaje y la investigación de carácter inter y transdisciplinario, para dar respuesta a los retos de la sociedad actual" (Universidad Javeriana, 2019, p. 1).

Planteamientos como los anteriores, se argumentan dado que se enmarcan en una dinámica impulsada por relaciones de orden político y económico que condicionan la autonomía de las universidades, pero también el diseño de propuestas curriculares, tales como: lógicas de acreditación, renovación de registros calificados, reformas educativas, fortalecimiento a la investigación. Aquí entran en juego discursos macro como desarrollo (desarrollo económico, desarrollo sostenible, desarrollo sustentable, desarrollo humano), capital humano, competencias, modernización, entre otros, que marcan unos derroteros claros frente a qué es importante enseñar, aprender $y$, por tanto, en qué formar. Así, por ejemplo:

se busca responder en lo educativo a las exigencias derivadas de los nuevos planteamientos de orden social, económico y político generados en el país con el advenimiento del nuevo milenio. Un asunto prioritario en el sentido anterior es la formación y aprendizaje permanente de los educadores de la región en la enseñanza de las ciencias naturales y en la educación ambiental, aspecto relevante y central de la articulación entre el desarrollo económico y el desarrollo científico de la nación. (Universidad del Valle, s.f., p. 1)

A partir de las relaciones que se evidencian, la formación se sitúa de la mano con el aprendizaje permanente, en cuanto condición para la constitución de sujetos competentes, talentosos y útiles a una sociedad que establece una serie de exigencias. Así, por ejemplo, se encuentra que

La formación continua es un imperativo en el ejercicio de la docencia [...] La velocidad de los cambios culturales y científicos así lo exige [...] La formación permanente debe propender hoy, no sólo a la apropiación de los saberes sino a la producción de los mismos. (Ministerio de Educación Nacional, MEN, 2005, p. 1)

Por tanto, la formación permanente es una necesidad que cambia los modos de relación del maestro con el mundo, consigo mismo y con el saber que enseña, pues

El propio saber experimentó una transformación capital al ser considerado cada vez más como una herramienta capaz de resolver problemas [...] un capital cuya función consiste en incrementar la capacidad humana de dominio de la naturaleza con el fin de hacerla servir más eficazmente al servicio del bienestar. El pueblo necesita ciertos conocimientos ligados a la práctica para su bienestar. Los demás, al ser inútiles, se devalúan. (Laval, 2004, pp. 38-39)

Se evidencia, entonces, una serie de discursos y prácticas que atraviesan la formación de maestros en general, estableciendo exigencias que instauran un deber ser alrededor de su profesión y de sí mismo, situando con ello una serie de saberes en los que sería importante formar, y enseñar, en el marco de la escuela, pues representan ciertas posibilidades y dotan a los sujetos de habilidades y competencias para enfrentarse a las necesidades de los diferentes contextos. Estos aspectos y relaciones no siempre han funcionado de la misma manera, por tanto, la mirada histórica que se propone permite develar otros elementos que entraron en juego en un momento determinado y que posibilitaron la formación de maestros de Biología desde otros presupuestos. Así las cosas, a continuación de despliegan algunos de estos aspectos que en su confluencia se convierten en condiciones de emergencia para la titulación de los primeros profesores de Ciencias Biológicas en el país.

\section{Las primeras facultades de Educación en Colombia: el posicionamiento de la formación de maestros}

Las condiciones de posibilidad relacionadas con la creación de las primeras facultades de Educación en Colombia y con las titulaciones de licenciados tienen 
que ver con el interés de fortalecer la formación de profesores a través de modernas metodologías pedagógicas, aumentar la población docente para atender los requerimientos de las escuelas, garantizar mejores condiciones laborales y establecer criterios rigurosos frente a quienes pueden ejercer el oficio, dado que se argumenta que la grave situación del maestro se debe a que muchas personas con el objeto de lograr un sustento se disponen a enseñar cualquier saber:

Os aseguro señoras y señores profesores que una de las causas más graves de la situación misérrima del profesorado es esa nube de gentes que, por hacer algo, por emprender en otro oficio o por no dejarse morirse de hambre ofrecen enseñanza por cualquier cosa. (López, 1936, p. 38)

Del mismo modo, se deja entrever la necesidad de formar profesionalmente a los docentes para reivindicar el ejercicio de su labor y, sobre todo, lograr construir una cultura académica frente a la docencia, pues se reconoce

El poco atractivo que tiene en Colombia la carrera del magisterio, lo que contribuye a que el personal normalista sea deficiente en general, pues es verdad sabida que no habiendo halago futuro, los estudiantes buscan los claustros de la normal como último recurso, y que muchos van sin la debida vocación. (Memoria del Ministro de Instrucción y Salubridad Públicas, 1924, p. 32)

Situación que se complejiza dado que las estadísticas muestran un gran número de maestros que ejercen la labor sin contar con título alguno, "todo esto hace ver la necesidad importantísima en que estamos de preparar convenientemente la cantidad tan creciente de maestros sin grado, aunque entre ellos, justo es reconocerlo, hay muchos que tienen la suficiente idoneidad adquirida en la práctica" (Memoria del Ministro de Instrucción y Salubridad Públicas, 1926, p. 16).

Para atender estas situaciones, el Ministerio, de acuerdo con las recomendaciones de la segunda Misión Alemana, concibe la idea de contratar un grupo de profesores normalistas extranjeros para establecer cursos de información entre los maestros que cuentan con una preparación deficiente. Estos cursos tienen una duración

de cinco meses para cada agrupación, y los cursos serán fijados por los técnicos teniendo en cuenta lo fundamental de la pedagogía moderna y el grado de conocimiento de que dispongan los grupos que vengan. Artes manuales, tan ignoradas por nosotros, jardines infantiles, pedagogía en todas sus formas, ciencias físico-químicas, aplicables en industria manual, serán las principales materias que se estudien. (Memoria del Ministro de Instrucción y Salubridad Públicas, 1926, p. 15)

Paralelamente, se desarrollan otros cursos complementarios. Así en 1932 se crea en Bogotá el Curso de Orientación Pedagógica, el cual estuvo a cargo de Agustín Nieto y tiene como propósito la formación para supervisores escolares. En 1933 se consolida el Curso de Información para maestras graduadas de Bogotá. Allí se destaca la profundización en materias como botánica, cultura general, enseñanza de la religión, práctica de la gimnasia, psicología experimental y la instrucción de ciegos y anormales (Jiménez y Figueroa, 2002). Se espera entonces que con el despliegue de estos escenarios, sumado al personal que se forma en las normales y cuyo perfeccionamiento se realiza en los institutos pedagógicos, se pueda contar con un magisterio preparado y capaz para la enseñanza de la juventud.

Ahora bien, entre 1926 y 1928, bajo las directrices del secretario de Educación Rafael Bernal Jiménez y del rector, el alemán Julius Síeber, se plantea la necesidad de formar los "maestros" para las escuelas normales, "los maestros de maestros". Así, en 1928 bajo la Ordenanza n. ${ }^{\circ} 10$ de la Asamblea Departamental de Boyacá, se consolida el Curso Suplementario de Especialización, cuya área de estudio inicial es Física y Química, formando docentes con la metodología de la enseñanza activa, y la disciplina de confianza, todo enmarcado en la investigación y el espíritu de superación (Universidad Pedagógica y Tecnológica de Colombia, UPTC, 2010). Posteriormente, el Decreto 150 de 1931 establece las ramas de especialización, entre las que se encuentra la naturalista-matemática, la pedagógica, la agrícola y la filológico-histórico-geográfica, con una duración de dos años cada una. La fuerza que toma el Curso Suplementario es tal que con base con este se enuncia

El Curso Suplementario o de Especialización que el Departamento de Boyacá ha sostenido en la Escuela Normal de Tunja, se reorganizará con la cooperación nacional hasta tomar el carácter de una Facultad de Pedagogía destinada a la preparación de profesorado para la enseñanza secundaria y a la instrucción normalista superior de que habla el artículo anterior. (Decreto 301, 1933, artículo 1)

En el marco de la Facultad en mención, se asume que la formación del profesorado de la enseñanza superior y normalista exige la preparación de la segunda enseñanza y cuatro años más de estudio en algunas de las siguientes especializaciones: Pedagogía, Lenguas Extranjeras, Matemáticas y Ciencias Fisicoquímicas, Ciencias Naturales y Castellano e 
Historia. El reconocimiento que alcanza la Facultad de Pedagogía con sus especializaciones lleva a expedir el Decreto 1379 de 1934 mediante el cual se crea la Facultad de Ciencias de la Educación (masculina) en Tunja, y que reglamenta los títulos de licenciado y de doctor; para este último, se exige una práctica de dos años, un examen de grado y la sustentación de una tesis. Así entonces:

Artículo $1^{\circ}$. Créase la Facultad de Ciencias de la Educación para hombres, en la ciudad de Tunja, como dependencia de la Escuela Normal de Institutores de esa ciudad, la cual funcionará y se considerará como parte de la Universidad Nacional, y estará, por lo tanto, bajo la dirección superior del Ministerio de Educación Nacional.

\section{$[\ldots]$}

Artículo $3^{\circ}$. Los alumnos que hayan recibido o recibieren el título de licenciados de la Facultad de educación de Tunja, podrán adquirir el grado de doctor en sus respectivas especialidades, previa comprobación de que el pénsum por ellos hecho es, tanto en extensión como en materias, el oficial, y previos los dos años de práctica reglamentarios.

(Decreto 1379, 1934)

La Facultad de Ciencias de la Educación de la Universidad Nacional (masculina), nombrada en el decreto anterior, se establece con los Decretos 10 de 1932 y 1990 de 1933, y tiene como objetivos:

primero, la preparación del personal directivo de las escuelas normales; segundo, la preparación del profesorado para la enseñanza de las distintas materias en los establecimientos secundarios y particularmente en las escuelas normales; tercero, la preparación de los inspectores escolares y de los maestros de escuela tipo; cuarto, el estudio y agitación de las cuestiones educativas en orden al establecimiento de los problemas que afecten los destinos históricos del pueblo colombiano. (Decreto 1990, 1933, artículo 2)

Esta comprende las siguientes secciones de estudio: Pedagogía, Ciencias Históricas y Geográficas, Ciencias Físico-Químicas, Biología, Filosofía y Letras, Matemáticas e Idiomas, con lo que se observa que, desde estas secciones comprendidas como escuelas de especialización, se despliega una apuesta por la formación de profesores en saberes y disciplinas específicas. Así la noción de especialización permite atender a "A). La complejidad, la diversidad y la extensión de los conocimientos en el estado actual de la ciencia. B). La conveniencia de ganar profundidad, consagrándose la atención a un solo campo de estudios" (Facultad Ciencias de la Educación de la Universidad Nacional, 1934, p. 41). No obstante, dada la situación fiscal y económica de la institución, se decide abrir solamente la sección de Pedagogía y la de Ciencias Histórico-Geográficas, pues

preferimos este ramo porque consideramos que los estudios históricos y geográficos van a llenar un vacío en nuestra preparación nacional, es decir, el conocimiento de nuestro territorio y de los factores constitutivos de nuestra nacionalidad, no ya en forma empírica y aislada, sino de acuerdo con una disciplina seria y alta que dé por resultado el basar la meditación de los problemas nacionales sobre una consideración geopolítica y el orientarlo hacia fines históricos definidos. (Bernal, 1933a, p. 198)

Los aspirantes a las secciones de la Facultad deben ser maestros graduados en escuelas normales oficiales o deben poseer el certificado de estudios de segunda enseñanza, también debidamente reconocido por el Ministerio de Educación Nacional, y deben presentar las pruebas reglamentarias para el ingreso ${ }^{2}$. Frente a las titulaciones, se establece que los estudios de cada una de las distintas escuelas de especialización se verificarán en tres años lectivos ordinarios, al cabo de los cuales, si el alumno ha aprobado todos los cursos reglamentarios, recibirá el título de licenciado en Ciencias de la Educación, con la especificación de la especialidad correspondiente. Del mismo modo, la Universidad Nacional otorga el título de doctor en Ciencias de la Educación a los licenciados que después de haber practicado durante dos años en el profesorado, normalista o secundario, bajo la inspección de la Facultad, soliciten examen de grado y lo sustenten satisfactoriamente, mediante presentación de estudio serio y personal de tesis (Decreto 1990, 1933).

2 Al respecto, se encuentra el Decreto 1487 de 1932, el cual plantea una reforma a la enseñanza primaria y secundaria. Allí se establece que los bachilleres que pretendan seguir la carrera del magisterio ingresarán a la Facultad de Educación, que comprenderá dos años de estudio para llegar a ser maestros de escuela primaria, y cuatro para profesores de segunda enseñanza. El aspirante a maestro de escuela primaria que hubiere cursado los dos años en la Facultad de Educación entrará a ejercer su profesión como maestro provisional por un año, terminado el cual recibirá el título de maestro graduado de primera categoría, si a juicio del jurado nombrado por el Gobierno tiene aptitudes físicas, mentales y morales para ejercer esta profesión. Para los actuales maestros graduados estará abierto en la capital de la República un Curso de Información que durará un año, terminado el cual, y mediante un examen, cuyas condiciones serán determinadas por el Gobierno, podrán los concurrentes a este curso recibir un certificado que los acredite para servir las principales direcciones de las escuelas primarias o para ejercer otros cargos de importancia en el ramo de la educación. 
Por otro lado, en sintonía con la creación de las facultades de Ciencias de la Educación en Bogotá y Tunja, de carácter masculino, Francisca Radke en compañía con el Consejo Directivo del Instituto Pedagógico Nacional para señoritas, solicita al Ministerio de Educación Nacional la creación de la Facultad de Ciencias de la Educación para Mujeres, argumentando la excelente preparación para profesoras aptas para la enseñanza didáctica y para la dirección e inspección de la enseñanza pública nacional con la que ha venido funcionando la institución. Por consiguiente, a través del Decreto 857 de 1934 "Créase la Facultad de Ciencias de la Educación para Mujeres como entidad dependiente del Instituto Pedagógico Nacional para señoritas, dentro del cual funcionará y se considerará como parte de la Universidad Nacional" (artículo 1).

Mediante el Decreto 95 de 1934, se especifican las primeras secciones de especialización para optar a la Licenciatura: Ciencias Físico-Químicas, Ciencias Biológicas, Matemáticas, Ciencias Pedagógicas y Gimnasia y Deportes, pero solo funcionan las especializaciones de Pedagogía, Matemáticas y Ciencias Biológicas. A la Facultad tienen acceso las maestras graduadas en escuelas normales oficiales, cuyos títulos estén aprobados por el Ministerio de Educación, con práctica de dos años, respaldado por el director de Educación Pública del Departamento respectivo. Los estudios se desarrollan en tres años y abordan las diferentes disciplinas científicas, metodología, pedagogía, psicología y prácticas de segunda enseñanza. No obstante, con base en el Decreto 1569 de 1934 se unifican los planes de las facultades universitarias oficiales, nacionales y departamentales. Por tanto, el plan de estudios de la Facultad de Ciencias de la Educación para señoritas sería el mismo que para las facultades masculinas o mixtas.

En términos generales se promulga una formación que le permita al maestro integrar las dimensiones de la pedagogía y las ciencias, pues se hace relevante:

formar un tipo de profesor que reúna a la vez la ciencia y la técnica de su transmisión; no quiere una ciencia sin pedagogía pero tampoco una pedagogía sin ciencia. Tampoco pretende formar y perfeccionar diletantes sin profundidad en ningún campo de saber, ni profesores eruditos en un miscroscópico sector de la ciencia. (Bernal, 1934, p. 715)

Así las cosas, con las prácticas que circulan desde las facultades de Ciencias de la Educación se comienza a posicionar la formación de maestros a nivel universitario, estableciendo con ello un estatus frente a la labor, abogando por el perfeccionamiento de la formación para la puesta en marcha de nuevas metodologías de enseñanza. Igualmente, se destaca que estas instituciones contribuyen a

a mejorar las condiciones socio-políticas de la población colombiana, puesto que se necesitaba mayor cobertura educativa [...] Las Facultades de Educación fueron instituciones formadoras de maestros que permitieron una evolución histórica y un cambio estructural desde las Escuelas Normales orientadoras de la educación y proyectadas a las Universidades Pedagógicas de Bogotá y Tunja en los años cincuenta (50) del Siglo XX. Las Facultades de Educación tanto de Bogotá como de Tunja, se concibieron como un aporte al desarrollo intelectual de los nuevos ciudadanos, formados en la cultura científica y pedagógica, cuyos fines respondieron a la solución de problemas de la educación nacional, a la cantidad de niños y jóvenes por fuera del sistema educativo, y a la falta de maestros y de personal directivo en la educación pública. (Figueroa, 2006, p. 18)

Sin embargo, la entrada en funcionamiento de estas instituciones y las marcadas diferencias entre licenciados y normalistas en cuanto a formación genera una serie de tensiones que se materializan mucho más desde la creación del escalafón nacional del magisterio. Así a través de la Ley 12 de 1934 se presentan las disposiciones para la formación del escalafón nacional del magisterio primario, y en correspondencia con la ley se encuentra el Decreto 1602 de 1936, por el cual se crea el escalafón del magisterio. De este modo se halla:

\begin{abstract}
El 19 de octubre de 1936, el Ministerio dictó la Resolución No. 358 clasificando en el escalafón a casi la totalidad del magisterio del país. A pesar de que el magisterio carecía de Normas que le garantizaran el derecho al ejercicio de su profesión, esta disposición no fue la de mayor aceptación por cuanto los beneficios alcanzados con ella sólo abarcaron el campo de lo secundario, dejando a un lado aspectos tan importantes como el establecimiento y el respeto a la carrera docente, es decir una verdadera profesionalización acompañada de un escalafón que garantizara sus derechos y asegurara su promoción. (Gómez et al., 1982, p. 145)
\end{abstract}

Sumado a ello, las cuestiones políticas y las influencias personales se constituyen en elementos decisivos frente al ascenso de los maestros, razón por la que se desconocen los criterios de idoneidad para el ejercicio de la labor, lo que genera indiscriminación y rechazo del magisterio. Por tanto, hacia finales de 1940 el Gobierno modifica la norma para hacerla más clara frente a los procedimientos de ascenso, clasificación y remuneración. 
Otro aspecto característico de la formación de maestros durante la época es el enfoque religioso católico que se cruzó con el desplazamiento de una educación tradicional hacia elementos de la Escuela Nueva. No obstante, son los liberales quienes impulsan una nueva visión alrededor de la formación con carácter laico y adscrita al Estado. Por tal motivo, el gobierno de López Pumarejo decide centralizar la formación superior de maestros a través del Decreto 1917 de 1935, donde se establece fusionar las tres facultades de Educación, dejando como punto central aquella que dependía de la Universidad Nacional y por tanto: "Los alumnos y alumnas de la Facultad de Educación de Tunja y del Instituto Pedagógico Nacional para señoritas que desearen continuar estudios en la Facultad de Educación de Bogotá harán solicitud de matrícula" (artículo 2).

Posteriormente, mediante la Ley 39 de 1936, se cambió el nombre de Facultad de Educación por el de Escuela Normal Superior: "La Facultad de Ciencias de la Educación continuará funcionando con el nombre de Escuela Normal Superior bajo la dirección inmediata del Gobierno y con independencia de la Universidad Nacional" (artículo 4). Dicho cambio se argumenta desde la posibilidad de vigilancia y control que el Ministerio tendría para reglamentar los programas y las condiciones de enseñanza. Así "la Facultad de Tunja tuvo una duración de 15 meses, la del Instituto Pedagógico de 17 y la de la Universidad Nacional de 24 meses" (Herrera y Low, 1994, p. 26).

De fondo estas transformaciones están atravesadas por fuertes disputas partidistas entre liberales y conservadores, las que giran alrededor del carácter laico o religioso que debe tener la educación, la pertinencia de una educación mixta o por sexo, la preservación de los valores familiares y culturales y la apertura ideológica. Estos elementos atravesaron los modos de comprensión de la educación y de la pedagogía, pues se evidencia el "tránsito de una pedagogía basada en la moral biológica propuesta por Ovidio Decroly [...] a una pedagogía activa, centrada en la moral social y conceptos sociológicos y culturales, impulsada por John Dewey" (Jiménez y Figueroa, 2002, p. 38). La pedagogía activa propuesta se sustenta desde una perspectiva pragmática y una mirada a la población desde la economía, la sociología, la antropología, entre otros saberes, que pretenden disciplinar a los sujetos para concretar las metas ligadas a la modernización e industrialización. Así se observa que la mirada frente a la educación se hace más desde unos presupuestos políticos y económicos, dejando en segundo lugar la preocupación por la pedagogía y la formación, aspecto que se evidencia desde la primera Conferencia Nacional del
Magisterio, al encontrarse que esta "se ocupa principalmente de aquellas cuestiones urgentes, referentes casi todas al aspecto orgánico de la educación pública, dejando para más tarde los problemas referentes a los aspectos puramente pedagógicos y metodológicos" (Bernal, 1933, p. 533).

Los sectores más tradicionales de la sociedad catalogaron como comunista, anticristiana y antimoral la tendencia ideológica dada a la educación desde una perspectiva liberal, acusando al Estado de ejercer un monopolio en la enseñanza; no obstante, desde Herrera (2012), se destaca que en el imaginario social se reconoce el periodo de gobierno liberal como una época que favorece los procesos de modernización, industrialización, urbanización, así como de apertura económica y social.

\section{E1 acontecimiento: los primeros licenciados en Ciencias Biológicas}

La titulación de los primeros licenciados en Ciencias Biológicas en Colombia es fruto del esfuerzo de los procesos de formación de las primeras facultades de Educación y de la necesidad de contar con profesionales de la educación que cumplan con los requerimientos para formar en saberes como la botánica, la higiene, la fisiología, la zoología, la evolución, entre otros, que resultan ser fundamentales para la época dadas las dinámicas de vida asociadas al cuidado, a los campos y la agricultura, pero también al impulso que comienza a recibir la ciencia.

En este contexto, la Facultad de la Universidad Nacional funciona entre 1933 y 1936, tiempo durante el que titula a 27 licenciados en Ciencias de la Educación; la Facultad de Tunja, teniendo en cuenta el Curso Suplementario legalizado en 1928, logra titular en 1934 a José Estiliano Acosta, Juan N. Segura y Florentino Cortés en el área de Matemáticas y Fisico-Química; a Antonio Perilla y Marcelino Páez en 1935 como Licenciados en Idiomas, y finalmente, en Pedagogía egresaron cinco licenciados: Eduardo Malagón, Max León, Luis Lizarazo, Moisés Melo y Rafael Celi (Ríos, 2006).

Ahora bien, atendiendo a que la Facultad de Ciencias de la Educación de Bogotá no logra poner en funcionamiento la especialidad de Biología, dados los problemas económicos por los que transita, se puede afirmar que las primeras licenciadas en Biología o en Ciencias Biológicas egresan de la Facultad de Educación de Mujeres en 1935, como lo sustentan Ríos (2006) y Gámez (1998) al retomar a Radke (1936), y que a través del Decreto 857 de 1934 se especifica 
que el Ministerio de Educación Nacional expedirá el título de licenciadas en su especialidad al grupo de estudiantes inscritas en el Instituto Pedagógico desde hace tres años, perfeccionando sus estudios en las ciencias físico-químicas y biológicas. De hecho así se hace, y en 1935 la Facultad de Ciencias de la Educación entrega cuatro diplomas de Licenciatura: dos en Ciencias Biológicas y dos en Ciencias Físico-Químicas.

Una licenciada de cada área, junto con dos profesoras más del Instituto, viajan a Alemania a adelantar algunos estudios, dos en ciencias y dos en educación física y trabajos manuales, respectivamente, con la metodología de Kerschensteiner. De esta manera, se continuaba en el exterior la preparación del cuerpo docente nacional, capaz de reemplazar a la Misión Alemana cuando terminara su labor (Gámez, 1998).

Con este acontecimiento, se comienza a posicionar la formación de maestros de Biología en el país, encontrando para el caso que dicha preparación se fortalece desde las apuestas de la Escuela Normal Superior, pues hacia 1936 se ofrece especializaciones en Pedagogía, Ciencias Geográficas, Ciencias Naturales, Idiomas y Matemáticas. Posteriormente, en 1938 con el Decreto 1218 se establece

La Escuela Normal Superior funcionará con las siguientes secciones: a) Ciencias sociales; b) Ciencias, físicas y matemáticas; c) Ciencias Biológicas y Químicas; d) Filología e idiomas, y e) Bellas artes, orientadas hacia la preparación de profesores para la enseñanza secundaria. (Artículo 1)

En relación con la titulación ${ }^{3}$ de la Escuela, se continua otorgando los grados de licenciado y doctor en Ciencias de la Educación. Para ser licenciado se exige haber cursado y aprobado todas las materias de la Especialización, tener una buena calificación en la práctica pedagógica y demostrar buena conducta:

El alumno que haya cursado y aprobado todas las materias del pénsum de la Escuela Normal Superior en la respectiva especialización, tendrá derecho a recibir el título de Licenciado en Filología e Idiomas, Ciencias Biológicas y Química, Ciencias Sociales

3 Frente a las titulaciones es importante tener en cuenta el Decreto 192 de 1951, en el cual se dictan algunas disposiciones frente a la educación normalista. Al respecto se enuncia que la Escuela Normal Universitaria de Bogotá confiere el título de licenciado en cada una de las especialidades que allí se cursen. Por otro lado, las escuelas normales superiores quedan autorizadas para conferir a los alumnos que aprueben los seis años del Plan de Estudio el título de Grado Superior de Maestro; las escuelas normales rurales, el de Maestro Rural y los colegios privados de orientación normalista aprobados por el Gobierno, el de Certificado de Competencia para el Magisterio Elemental. y Economía, Matemáticas y Física, Ciencias de la Educación, Educación Física, etc., según el caso. (Decreto 163, 1948, artículo1)

El título de doctor se otorga a aquellos licenciados que han ejercido la enseñanza mínimo dos años y presentan una tesis apoyada en una investigación científica original. Para 1940, se hallan algunos planes de estudio correspondientes a la formación inicial de maestros en el área de ciencias, particularmente de las secciones de biología y química, los que se

caracterizan por ser un listado de asignaturas cada una de las cuales se impartía semanalmente, como se detalla a continuación [...] CIENCIAS BIOLÓGICAS Y QUímICAS. Primer año. Histología (técnicas vegetal y animal) 2h/s; Física biológica (curso completo) 4h/s; Geología (general y particular de Colombia) 2h/s; Química (metaloides y metales) 4h/s; Dibujo y modelado (aplicado a las ciencias biológicas) 2h/s. Segundo año. Botánica (Anatomía y Fisiología) 4h/s; Zoología (invertebrados) 4h/s; Química (metales continuación) 4h/s. Tercer año. Botánica (taxonomía) 4h/s; Zoología (vertebrados) 4h/s: Química (del carbono) 4h/s. Cuarto año. Anatomía y fisiología humanas, 6h/s; Biología, 2h/s; Química biológica, 4h/s. (Pérez et al., 2009, p. 48)

En términos de lo pedagógico, es posible rastrear DE LO PEDAGóGICO Y DIDÁCTICO [...] se encontró que todos los profesores en formación inicial, de las seccionas en que se dividía la Escuela Normal Superior de Colombia tenían que cursar asignaturas correspondientes a cultura general y pedagogía, así: Primer año: Psicología general 2h/s; Antropología infantil 2h/s; Higiene escolar 2h/s; Estadística cultural y pedagógica $2 \mathrm{~h} / \mathrm{s}$; Inglés $2 \mathrm{~h} / \mathrm{s}$. Segundo año: Pedagogía sistemática 2h/s; Psicología infantil y de la adolescencia 2h/s; Prácticas metodológicas, 2h/s; Historia de la filosofía (filósofos antiguos y modernos y lógica y teoría del conocimiento) 3h/s. Tercer año: Pedagogía (aplicada a la segunda enseñanza) 2h/s Psicotecnia (orientación profesional) 1h/s; Prácticas metodológicas 4h/s; Historia del arte 3h/s. Cuarto año: Metodología 2h/s; Prácticas metodológicas 4h/s; Nmosiquiatría infantil 2h/s; Geografía (económica y social de Colombia) 2h/s. (Pérez et al., 2009, p. 49)

Desde este acercamiento, se observa que la biología y los diversos saberes ligados a ella se posicionan con mayor fuerza desde la formación de maestros. Así la Escuela Normal se caracteriza por ser

la institución en la que por primera vez en la historia de las ciencias se reconoce la biología como una ciencia; algo en lo que se adelanta a las reflexiones respectivas (MAYR, 1998; 2006). Para reiterarlo, 
en Colombia los programas académicos para la formación de físicos y biólogos, corresponden a la segunda mitad del siglo xx. Es el Maestro Socarrás a quien se le debe la apertura de las licenciaturas en Biología y Química y en Física y Matemáticas, cuyos propósitos fueron introducir a los colombianos y colombianas en esos procesos que habían cambiado la concepción de mundo. (Pérez et al., 2009, p. 46)

En sintonía con ello, otra regularidad que se comienza a identificar es la relación que los planes de estudio establecen entre la biología y la química para la comprensión de la vida, posicionando metodologías de trabajo conjuntas. Asimismo, se evidencia que las cuestiones ligadas a la higiene se abordan ahora en clave de la formación pedagógica, no desde la historia natural como se visibiliza anteriormente.

El estudio de la ciencia se ve fuertemente impulsado dado que se procura la formación de maestros tanto en lo científico como en lo didáctico, promoviendo el aprendizaje a través de seminarios y de investigación. Además de ello, se cuenta con una planta docente extranjera fuertemente capacitada y con diversos institutos anexos que favorecen las prácticas en general:

Según la filosofía de la Escuela Normal Superior, el corazón de la formación de un profesor, debe ser la investigación científica. Un profesor no puede formar las nuevas generaciones, si no está investigando en una ciencia determinada; así mismo, no se concibe ninguna especialización, sin los Institutos de Alta Investigación Científica. Así se creó el Instituto Etnológico Nacional, con la organización que dio el etnólogo Paul Rivet. También la Escuela de Altos Estudios Sociales y auspició también el Instituto Caro y Cuervo, en donde se forman los investigadores filólogos. También se creó el Instituto de Psicología Experimental, bajo la orientación del Maestro José Francisco Socarrás. El Instituto Nicolás Esguerra, un colegio de bachillerato de carácter experimental se convirtió en el centro de Práctica docente. (Ocampo, 1998, p. 191)

Bajo estas posibilidades, en la Escuela Normal Superior se busca la construcción de conocimientos vivos en tanto permiten acercarse a la realidad del país. Así entonces se habla de conocimientos para la vida, los que son construidos desde los laboratorios y las excursiones, pues toda enseñanza se hace desde el método de problemas y proyectos, retomando así a Dewey para reformar un sistema pasivo tradicional (Ospina, 1984).

Por otro lado, la formación en la Escuela Normal Superior plantea interrogantes alrededor de la preparación de los profesores para la enseñanza en la
Universidad, pues si bien el licenciado egresado de la Escuela Normal se capacita para desempeñar sus funciones en la enseñanza secundaria, no es suficiente para que asuma satisfactoriamente cátedras universitarias, dado que estas exigen un nivel superior de formación. Se reconoce entonces que en el país no existe ninguna institución destinada especialmente a la preparación de profesores de universidad. Por tanto, a través del Decreto 1575 de 1952, se dispone que la Escuela Normal debe crear los estudios de posgraduados con la finalidad de formar el profesorado para las escuelas normales universitarias y similares del país:

\begin{abstract}
El título que se otorgue será el de Profesor Habilitado, o sea, la "facultas docendi" para cátedras universitarias y lo otorgará la Escuela Normal Universitaria y los aspirantes que hayan hecho los estudios correspondientes en las siguientes ramas de la enseñanza: Pedagogía, Filosofía, Filología, Sociología, Física y Matemáticas, Química, Biología y las demás que se establezcan por exigencias del desarrollo cultural y económico del país. (Decreto 1575, 1952, artículo 2)
\end{abstract}

Podrán ingresar a dichos estudios quienes posean el título de licenciado o el de doctor expedido por las normales universitarias o por alguna otra de las universidades o facultades oficialmente reconocidas. Los estudios se desarrollan en cuatro años, dos de ellos se cursan en la Escuela Normal Universitaria y los otros dos en una universidad del exterior señalada al estudiante por la Normal Universitaria.

Por estos y otros logros, la Escuela Normal Superior destaca por constituirse en el escenario que promueve el avance de distintas áreas de saber, el fortalecimiento de la formación de maestros a nivel universitario en diversas especialidades, la creación de otras instituciones en apoyo al logro de sus propósitos formativos, contribuyendo con ello al desarrollo del país. No obstante, dada la crisis de finales de la década de 1940, más las tensiones políticas, religiosas y económicas entre conservadores y liberales, sumado al "recorte gradual del presupuesto, hasta las hostilidades personales hacia sus profesores y alumnos, a los contenidos que allí se enseñaban y a la orientación pedagógica que la inspiraba" (García, 2004, p. 3), se consideraron condiciones para el cierre de esta institución, situación que se materializa en 1951 con el Decreto 1995:

Artículo $1^{\circ}$ La Escuela Normal Universitaria se dividirá en lo sucesivo en sus secciones masculina y femenina, así: la primera se llamará Escuela Normal Superior de Varones, y desde el $1^{\circ}$ de enero de 1952 funcionará en el local que ocupa la 
Escuela Normal de Varones de Tunja; la segunda, en el Instituto Pedagógico Nacional, que se eleva a la categoría de Instituto Pedagógico Nacional Superior y tendrá las características de Escuela Normal Superior Femenina. Las denominadas Escuelas Normales Superiores de llamarán simplemente Escuelas Normales.

Mediante el Decreto 2655 de 1953 y el Decreto 547 de 1954, la Escuela Normal Superior de Varones y el Instituto Pedagógico Nacional Femenino se transformarán, respectivamente, en Universidad Pedagógica de Colombia con sede en Tunja y Universidad Pedagógica Femenina en Bogotá. Esta última puede ser considerada una condición para la actual Universidad Pedagógica Nacional, donde se van a materializar las condiciones para el despliegue de la Licenciatura en Biología de manera formal hacia 1968 y sus distintas versiones hasta la actualidad.

\section{Otras reflexiones}

Rastrear las huellas de la formación de maestros de Biología en el país surge de una pregunta por la propia práctica como profesora de dicha disciplina, la que se entrecruza con las tensiones y los retos que implica ser maestro hoy. Este ejercicio evoca el pensamiento y la pedagogía en la medida que sitúa un interrogante por los modos de constitución de los maestros, las transformaciones y los desplazamientos que los atraviesan, lo cual permite situar la formación como un devenir, donde el sujeto hace y construye desde su experiencia y donde la enseñanza es la oportunidad de configurar una serie de problematizaciones frente a sí mismo.

Desde estos elementos, es posible enunciar que la emergencia y los modos de funcionamiento de la formación de maestros de Biología dan cuenta de ciertas regularidades y enunciados, tales como desarrollo, vida, gestión, regulación, población, salud-enfermedad, entre otros, que se mantienen a lo largo del tiempo, pero que las prácticas que despliegan adquieren cierta especificidad por las condiciones históricas que los atraviesan; de allí la importancia de este acercamiento.

Finalmente, los aspectos evidenciados y los cuestionamientos frente a la actualidad de los maestros de Biología pueden sentar varias inquietudes alrededor de su porvenir y marcar algunos derroteros para seguir investigando: ¿En qué biología nos formamos los maestros de esta disciplina? En el marco de la formación profesional de maestros de Biología, ¿se enseña la disciplina escolar?, ¿cuáles son las relaciones entre la biología como ciencia y la enseñanza de la biología?, ¿estamos asistiendo a la desaparición de la formación de maestros de biología?, ¿cómo se reactualizan estos programas?

\section{Referencias}

Bernal, R. (1933). Revista Educación. Órgano de la Facultad de Educación de la Universidad Nacional. Año 1. N. 4. Bogotá(4).

Bernal, R. (1934). La Facultad de Ciencias de la Educación. Educación, (17), 713-717.

Decreto 150 de 1931. Reglamento y Pénsum del Curso de Especialización Normalista. Revista Cultura.

Facultad de Ciencias de la Educación de la Universidad Nacional. (1934). Estatuto, Reglamento y Plan de Estudios. Editorial ABC.

Figueroa, C. (2006). Orígenes, formación y proyección de las Facultades de Educación en Colombia 1930-1954. Revista Historia de la Educación Latinoamericana, 4(8), 201-220.

Gamez, R. (1998). La formación docente en la obra de la doctora Ingrid Müller de Ceballos [tesis de maestría, Universidad de Antioquia].

García, N. (2004). Facultad de Educación: 50 años abriendo caminos. Memoria. Protagonista y testigo. Universidad de Antioquia. http://www.udea.edu.co/wps/wcm/connect/udea/b59be2f6-8f33-4428-9f76-f525747d2a26/ facultad-educacion-50anos-abriendo-caminos-educacion.pdf?MOD=AJPERES\&CVID=kQRgNvP

Gómez, O., Gómez, S. y Urrego, I. (1982). La educación en Colombia en el siglo XX. 1900-1980. [Tesis de maestría, Universidad de Antioquia].

Herrera, M. y Low, C. (1994). La Escuela Normal Superior. Universidad Pedagógica de Colombia.

Herrera, M. (2012). La Escuela Normal Superior 1936-1951: avatares en la construcción de un proyecto intelectual. En O. Zuluaga, Historia de la educación en Bogotá (tomo II, pp. 95-132). IDEP.

Jiménez, A. y Figueroa, H. (2002). Historia de la Universidad Pedagógica Nacional. Universidad Pedagógica Nacional.

Laval, C. (2004). La escuela no es una empresa. Ediciones Paidós.

López, J. (1936). La familia, el estado y la iglesia en la educación. Comentarios a algunos artículos del Proyecto de Nueva Constitución Nacional. [Estudio para optar al Grado de Doctor en Derecho y Ciencias Políticas, Universidad de Antioquia]. 
Maldonado, C. (2012). De la ciencia a la filosofía y viceversa. Cuestiones de Filosofía, (9), 71-86. https://doi. org/10.19053/01235095.627

Memoria del Ministro de Instrucción y Salubridad Públicas al Congreso. (1924). Imprenta Nacional.

Memoria del Ministro de Instrucción y Salubridad Públicas al Congreso. (1926). Imprenta Nacional.

Ministerio de Educación Nacional. (1932, 07 de enero). Decreto 10 de 1932, Por el cual se dictan algunas disposiciones sobre escuelas normales departamentales y se funda la Facultad de Educación. http://www.suin-juriscol.gov.co/viewDocument.asp?id=1002474

Ministerio de Educación Nacional. (1932, 13 de septiembre). Decreto 1487 de 1932, Sobre reforma de la enseñanza primaria y secundaria. http://www.suin-juriscol. gov.co/viewDocument.asp?ruta=Decretos/1296252

Ministerio de Educación Nacional. (1933, 05 de diciembre). Decreto 1990 de 1933. Por el cual se dictan disposiciones orgánicas de la Facultad de Ciencias de la Educación. http://www.suin-juriscol.gov.co/viewDocument. asp?ruta=Decretos $/ 1382172$

Ministerio de Educación Nacional. (1933, 13 de febrero). Decreto 301 de 1933. Por el cual se dictan disposiciones orgánicas relativas a la Escuela Normal de Institutores de Tunja. http://www.suin-juriscol.gov.co/viewDocument. asp?ruta=Decretos $/ 1069185$

Ministerio de Educación Nacional. (1934, 21 de abril) Decreto 857 de 1934. Por el cual se dictan disposiciones relacionadas con el Instituto Pedagógico Nacional para señoritas. http://www.suin-juriscol.gov.co/viewDocument.asp?id=1728008

Ministerio de Educación Nacional. (1934, 05 de julio). Decreto 1379 de 1934. Por el cual se dictan algunas disposiciones orgánicas relativas a la Escuela Normal de Institutores de Tunja, Facultad de Ciencias de la Educación. http://www.suin-juriscol.gov.co/viewDocument. asp?id=1739954

Ministerio de Educación Nacional. (1934, 02 de agosto). Decreto 1569 de 1934, Por el cual se fija el plan de estudios que regirá para las Facultades. Universitarias oficiales nacionales y departamentales. http://www. suin-juriscol.gov.co/viewDocument.asp?id=1310301

Ministerio de Educación Nacional. (1934, 17 de diciembre). Ley 12 de 1934, Por la cual se reorganiza el Ministerio de Educación Nacional y se dictan otras disposiciones sobre instrucción pública. Ministerio de Educación Nacional. http://www.suin-juriscol.gov.co/viewDocument. asp?ruta=Leyes $/ 1568122$
Ministerio de Educación Nacional. (1935, 25 de octubre). Decreto 1917 de 1935, Por el cual se reúnen en una sola las Facultades de Educación. http://www.suin-juriscol. gov.co/viewDocument.asp?id=1370272

Ministerio de Educación Nacional. (1936, 21 de febrero). Ley 39 de 1936. Por la cual se crea en el Ministerio de Educación Nacional la Sección de Publicaciones, se autoriza el envío de profesores universitarios al Exterior y se dictan otras disposiciones en el ramo de Educación. http://www.suin-juriscol.gov.co/viewDocument. asp?id=30036395

Ministerio de Educación Nacional. (1936, 06 de julio). Decreto 1602 de 1936, Por el cual se dictan disposiciones en desarrollo de las Leyes 12 de 1934 y 17 de 1935. http://www.suin-juriscol.gov.co/viewDocument. asp?ruta=Decretos $/ 1316678$

Ministerio de Educación Nacional. (1938, 09 de julio). Decreto 1218 de 1938. Por el cual se fija el pensum y el plan general de estudios para la Escuela Normal Superior. http://www.suin-juriscol.gov.co/viewDocument. asp?id=1245571

Ministerio de Educación Nacional. (1948, 25 de enero). Decreto 163 de 1948. Por el cual se reforma el Decreto número 1990 de 1933, sobre títulos de la Escuela Normal Superior. https://normograma.info/men/docs/ decreto_0163_1948.htm

Ministerio de Educación Nacional. (1951, 18 de septiembre). Decreto 1955 de 1951. Sobre educación normalista. https://www.mineducacion.gov.co/1759/articles-102876_archivo_pdf.pdf

Ministerio de Educación Nacional. (1952, 01 de julio). Decreto 1575 de 1952, Por el cual se establece y reglamenta la formación del profesorado universitario para las Escuelas Normales Universitarias y similares. Nacional. https://www.mineducacion.gov.co/1759/w3-article-103192.html?_noredirect=1

Ministerio de Educación Nacional. (1951, 30 de enero). Decreto 192 de 1951, Por el cual se adopta el plan de estudios para los Institutos de Enseñanza Pedagógica, y se dictan otras disposiciones sobre Educación Normalista. http://www.suin-juriscol.gov.co/viewDocument. asp?id=1710197

Ministerio de Educación Nacional. (1953, 10 de octubre). Decreto 2655 de 1953, Por el cual se crea la Universidad Pedagógica de Colombia, con sede en la ciudad de Tunja y se dictan otras disposiciones. https://www.mineducacion.gov.co/1621/articles-103443_archivo_pdf.pdf 
Ministerio de Educación Nacional. (1954, 04 de mayo). Decreto 547 de 1954. Por el cual se crea la Universidad Pedagógica Femenina independiente de la Universidad Pedagógica de Colombia.

Ministerio de Educación Nacional (MEN). (2005). Tres miradas a la formación docente. Altablero, (35). https:// www.mineducacion.gov.co/1621/article-89943.html

Ocampo, J. (1998). Los orígenes de las universidades pedagógicas en Colombia. Revista Historia de la Educación Colombiana, 1(1), 183-197. https://revistas.udenar.edu. co/index.php/rhec/article/view/1220

Ospina, J. (1984). La Escuela Normal: círculo que se cierra Boletín Cultural y Bibliográfico, 21(2), 3-16.

Pérez, R., Gallego, R. y Rincón, L. (2009). Estudios sobre la historia social de las ciencias y origen de la Escuela Normal Superior de Colombia. Studiositas, 4(2): 39-53.

Radke, F. (1936). Historia del Instituto Pedagógico Nacional para señoritas desde 1927 hasta 1935. El Gráfico.

Ríos, R. (2006). Las ciencias de la educación en Colombia: una investigación histórica sobre su proceso de institucionalización y apropiación en el saber pedagógico colombiano, 1926-1954. [Tesis de doctorado, Universidad de Antioquia].
Rodríguez, M. (2019). Bioeconomía y ciencias de la vida. Ludus Vitalis, 23(51), 201-205.

Ruiz, R. y Velázquez, B. (2014). La biología del siglo xxI: Innovación, bioética y bioarte. En M. A. González (coord.), Pròs bíon. Reflexiones naturales sobre arte, ciencia y filosofía (pp. 519-544). Editorial Universidad Nacional Autónoma de México.

Universidad del Tolima. (2016). Licenciatura en Ciencias Naturales y Educación ambiental. Colombia. http://idead.ut.edu.co/index.php/programas/ programas-pregrados/area-de-licenciaturas / licenciatura-educacion-ambiental

Universidad Javeriana. (2019). Licenciatura en Ciencias Naturales. https://www.javeriana.edu.co/ licenciatura-ciencias-naturales-educacion-ambiental

Universidad del Valle. (s.f.). Programa Licenciatura en Educación Básica con énfasis en ciencias naturales y educación ambiental. Colombia. http://iep.univalle. edu.co/licenciatura-en-ciencias-naturales-y-educacion-ambiental

Universidad Pedagógica y Tecnológica de Colombia (UPTC). (2010). Cartilla resumen del informe ejecutivo Autoevaluación con fines de acreditación Programa de Biología. 
